\title{
The effect of electrical passive cycling on spasticity in war veterans with spinal cord injury
}

\section{Seyed Mansoor Rayegani ${ }^{1}$ *, Hadi Shojaee ${ }^{2}$, Leyla Sedighipour ${ }^{1}$, Mohammad Reza Soroush $^{2}$, Mohammad Baghbani ${ }^{2}$ and Omm'ol Banin Amirani ${ }^{2}$}

1 Department of Physical Medicine and Rehabilitation, Shohada Hospital, Shahid Beheshti University of Medical Sciences, Tehran, Iran

2 Jaanbazan Medical Engineering Research Center, Tehran, Iran

\section{Edited by:}

Saumyajit Basu, Park Clinic, India

Reviewed by:

Harvinder Singh Chhabra, Indian

Spinal Injuries Centre, India

Shankar Acharya, Sir Gangaram

Hospital, India

\section{${ }^{*}$ Correspondence:}

Seyed Mansoor Rayegani, Professor of Physical Medicine and

Rehabilitation, Department of Physical

Medicine and Rehabilitation, Shohada Hospital, Shahid Beheshti University of Medical Sciences, Tehran, Iran. e-mail: rayegani@gmail.com

\begin{abstract}
Introduction: Muscle atrophy, spasticity, and deformity are among long term complication of spinal cord injury (SCl) veterans. There are numerous studies evaluating effect of functional electrical stimulation on muscle properties of $\mathrm{SCl}$ people, but less research has focused on the benefits of passive cycling in the management of spasticity and improving ROM of lower limbs in individuals with SCI. Aims: To evaluate the effect of electrical passive cycling on passive range of movement spasticity and electrodiagnostic parameters in $\mathrm{SCl}$ veterans. Methods: Sixty-four SCl veterans referred to two clinical and research center in Tehran were recruited in this prospective clinical trial. The subjects were divided into two groups according to electrical passive cycling usage: (1) patients who did not use pedal exercise (control group), (2) patients used Electrical passive cycling up to optimal level (intervention group). Main outcome measures included hip, knee, and ankle range of motion, spasticity scale, and electrodiagnostic parameters including F-Wave Consistency, F-Wave Amplitude, H/M Ratio, F/M Ratio, H-Reflex Onset Latency, and H-Reflex Amplitude. Data were recorded the time of receiving and 1 year after pedal exercise usage. Results: Sixtyfour $\mathrm{SCl}$ patients including $95.3 \%$ male, $4.7 \%$ female with mean age 43 years old were included in this study. All patients except one suffered from complete $\mathrm{SCl}$. The involved spinal levels were cervical (17.2\%), upper thoracic (34.4\%), lower thoracic (45.3\%), and lumbar (3.1\%). Spasticity scale decreased significantly after passive cycling in group 2. Also hip, knee, and ankle ROM in group 2 were significantly improved after pedal exercise. There was a significant difference in $\mathrm{H}$ max/M max (RT\&LT) and F/M ratio after versus before electric passive cycling system in group 2. Conclusion: These findings suggest that passive rhythmic leg exercise can lead to decrease in spasticity, increase in passive ROM of lower limbs and improvement in electrodiagnostic parameters of spasticity in patients with $\mathrm{SCl}$.
\end{abstract}

Keywords: spinal cord injury, spasticity, electrical passive cycling

\section{INTRODUCTION}

Spinal cord injury (SCI) is one of the critical and devastating injuries. As time grows, SCI causes widespread disability and dysfunction (Braddom, 2007). During the early part of the twentieth century, there was little progress made in the management of SCI, and most persons with SCI died within weeks or months. Nowadays due to medical and Rehabilitation development, life expectancy has grown as well (McArdle et al., 1994; Braddom, 2007; Frontera, 2008). As a consequence of relative sedentary or complete inactivity life style, these patients encounter muscle atrophy, joints stiffness, spasticity and deformity, cardiopulmonary sequelae, and other systemic complications. Any of the above mentioned phenomenon intensifies their disability (Daivis, 1993; Frontera, 2008), and lead to decreased quality of life and significant medical costs. Below the level of a SCI, the paralyzed muscles become atrophied It is considered that physical exercises on a continuous and precise basis maintains muscle tone both in trunks and the limbs, leading to prevention of joint contracture, maintenance of flexibility in joints and muscles and enhancement of the internal organs functions (Braddom, 2007; Devillard et al., 2007).
Physiotherapy, mechanical, and electrical equipments are usual methods prescribed for performing physical exercise in spinal cord injured patients, but they are time consuming and costly (Sköld et al., 2002; Duffell et al., 2008; Szecsi and Schiller, 2009). In recent years, new methods are introduced to help patients conduct physical exercises independently at home and this has been an evolution and a great progress. Electrical passive pedaling systems (EPPS) are among these innovated rehabilitation technologies by which the handicapped patients practice physical exercises independently (Therese et al., 2008). Passive rhythmic exercises entails several physical effects (Mutton et al., 1997). The main aim of passive movements of the legs is to preserve full range of motion over the joints that are immobilized and claim to have an effect on spasticity (Kakebeeke et al., 2005).

Even though there are numerous studies evaluating effect of functional electrical stimulation (FES) on muscle properties of SCI people, less research has focused on the benefits of passive cycling in the management of spasm and improving ROM of lower limbs in individuals with SCI (Sköld et al., 2002; Duffell et al., 2008; Therese et al., 2008). Improvements have been reported in 
spasticity (Kakebeeke et al., 2005), cardiac output and stroke volume (Therese et al., 2009), and muscle atrophy after a program of passive cycling (Therese et al., 2008).

Even though, SCI veterans are not suffering from geriatric sequelae, their physical condition and sedentary life style following the spinal injury makes cardiopulmonary disorders in veterans expectable. During active physical exercises, e.g., using EPPS, there will appear tachycardia, hypertension, and headache. Therefore, the exercise duration should be decreased and then again increased but gradually or even in some cases, unfortunately, quit the exercise. And this is one of several factors upon which we decided to conduct a research focusing on the usage method of EPS in veterans.

Furthermore; EPPS or electrical passive cycling has recently been applied for physical therapy in veterans; and as its effect on joints spasticity and electrodiagnosis parameters is not yet defined. Physical effects of EPPS usage in Iranian veterans with SCI had been studied in this trial and both objective and subjective signs in relation with EPCS/EPPS were assessed.

\section{MATERIALS AND METHODS \\ PATIENTS}

The present trial is a prospective clinical research performed on 64 SCI veterans.

According to the study method, all the SCI Veterans living in Tehran were invited to receive the Electric Passive Cycling equipment and to undergo clinical and electrodiagnostic tests by the assigned PM and R MDs of Shohada Hospital and JMERC (Janbazan Medical Engineering Research Center) which is affiliated to The Health deputy of the Foundation of Martyrs and Veterans Affair.

The criteria upon which the subjects recruited were as per follow:

a. The participant should be with SCI and provided with the electric passive cycling system.

b. The Veteran should be willing to participate and cooperate in the study,

Exclusion criteria:

1. Lower limb fracture.

2. Any other orthopedic disease in lower limbs.

3. Inappropriate collaboration during the course of trial.

Before the initiation of the trial, written consent had been signed by each of the participants with above criteria.

\section{STUDY DESIGN}

In this randomized prospective clinical trial, 74 veterans were recruited during 2 years. After being visited by the PM\&R MD and filling up the questionnaire of the veterans plan, they were provided with the required introductory letter to the delivering division temporarily established in the Research Center of Janbazan (veterans).

At the time, a simple randomization was performed, all patient with inclusion criteria were allocated by a random numbers and by using random number table to two groups: intervention (passive cycling) and control group (physical therapy). Equal allocation between two treatment arms were considered. Type of design was parallel group.

Routine physical therapy including stretching, ROM, and strengthening exercises were considered for control group. Thirtyseven veterans selected in control and intervention group during study period which was 2 months, eight patients from control were dropped from the study because they did not participate in physical therapy program regularly. Two patients from intervention group were dropped from the study due to inaccurate and irregular use if EPPS (Figure 1). Finally 64 patients completed the study and their data was analyzed.

\section{INTERVENTION GROUP}

Intervention group were placed in their own wheelchair in front of a motorized cycle (THERA-vital, Medica Medizin GmbH, Hochdorf, Germany). The veteran's legs were passively moved in a standardized way by that motorized cycle for required period of time.

The method of exercise with the pedaling system were taught to the veterans, its probable risks and benefits, and the features of this research plan.

Gradual increase in the length of exercise to the target level of $20 \mathrm{~min} / \mathrm{set}$ repeating three times a day was a guideline to be performed for 2 months.

Hence; SCI veterans were divided into two groups regarding cycling usage:

Group 1 (Control group) The veterans who did not use EPPS but just receive physical therapy for 2 months. (29 veterans); Group 2 (Intervention group) The veterans who used EPPS optimally/up to optimal level (35 veterans).

All patients were permitted to continue their previously activities including standing and walking with braces but were not permitted to participate in non-study-related lower extremity repetitive motion tasks or electrically stimulated exercise.

All veterans in intervention group signed informed consent forms, which was approved by the governing institutional review board.

\section{DATA COLLECTION}

Demographic information was obtained by interviewing with the patients. All data including clinical examination results, goniometric parameters, and electrodiagnostic findings (before and after intervention) were recorded in patients' files.

\section{CLINICAL DATA}

Physical examination data, e.g., muscle strength (according to Kondal Scale; Braddom, 2007), level of SCI, spasticity scale [Ashworth et al. (2004) Modified, were obtained by Physical Medicine and Rehabilitation (PM\&R) MD through examinations and observations. All examinations and tests had been performed by two assigned PM\&R MD].

In order to measure the passive range of motion in joints, Goniometer was utilized. 


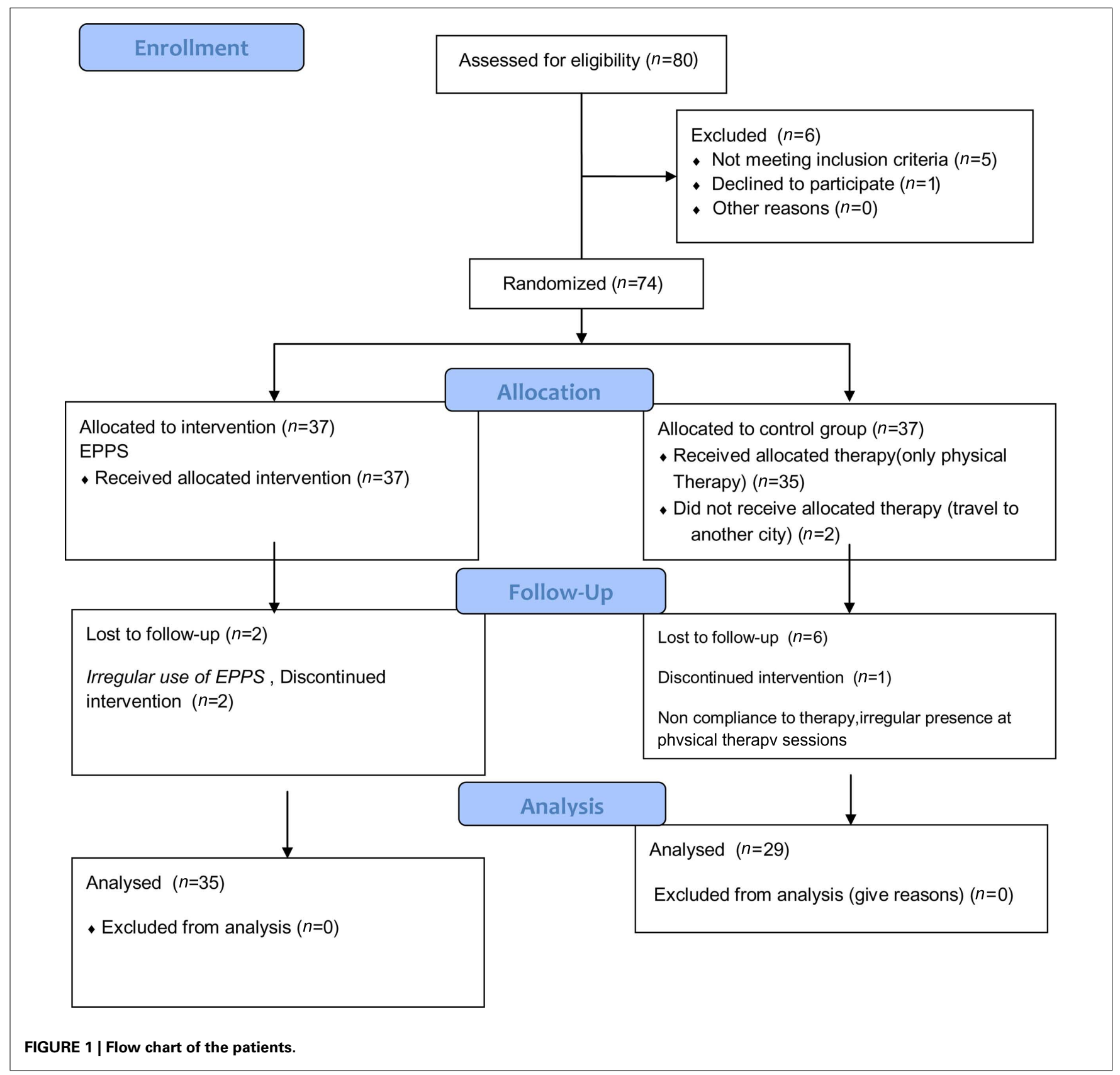

\section{ELECTRODIAGNOSIS}

Electrodiagnostic tests were conducted via a pair of two-channeled TOENNIES-PLUS, German made devices in the research and academic center. Electrodiagnosis was performed and reported by a PM\&R MD.

\section{OUTCOME MEASUREMENT}

The objective signs evaluated in veterans were as per follow: (1) Hip, knee, and ankle passive range of motion, (2) spasticity scale (according to Modified Ashworth Scale).

All clinical assessments were performed three times during the study: (1) at the first day of study, (2) 2 months later after finishing study protocol (passive cycling in group 1 and physical therapy in group 3) 1 year after finishing study protocol.

Finally, the results of clinical evaluations performed 1 year after finishing the study protocol were reported.

\section{ELECTRODIAGNOSTIC PARAMETERS}

We performed the electrodiagnostic tests by a two channel synergy electrodiagnostic instrument

- H-Reflex (LT\&RT), Amplitude - Change

- $\mathrm{H}(\max ) / \mathrm{M}(\max ), \mathrm{H}(\max ) / \mathrm{M}(\max )$ - change, H-Reflex (RT\&LT), Onset Latency - Change 
- F - Wave - (RT\&LT) - Consistency - Change, F-Wave (RT\&LT), Amplitude - Change.

\section{F/M RATIO (RT\&LT) CHANGE}

The variables mentioned above had been measured three times during the trial: (1) at the first day of study (the same day that subjects were recruited), (2) 2 months later after finishing study protocol (passive cycling in group 1 and physical therapy in group (3) 1 year after finishing the period of the study, we commenced another series of electrodiagnostic tests in both associated centers and in both two groups with different therapy protocol. Each subject was examined by the exact PM\&R MD who had visited them in the first phase last year.

Hence, the electrodiagnostic tests (at the first day of the study and after 1 year) were performed at the fixed time for all subjects.

Finally, the results of electrophysiologic tests performed 1 year after finishing the study protocol were reported.

\section{DATA ANALYSIS}

In order to analyze the data resulted from the study, we utilized Paired $t$-test, Single way Analysis of Variance, and Kruskal Wallis test. $P<0.05$ was considered as the significance level.

\section{RESULTS}

\section{PRE-INTERVENTION DATA}

The participants included $93.5 \%$ male veterans and $4.7 \%$ female ones. The mean age of patients was 43 years old, while most of the cases ( 21 persons $=32.8 \%$ ) had become SCI during 1985-1986.

All patients except one suffered from complete SCI. The involved body area included cervical injury in 11 subjects $(17.2 \%)$, upper thoracic injury in $22(34.4 \%)$, and lower thoracic injury in $29(45.3 \%)$, and lumbar spinal injury in two patients $(3.1 \%$; Table 1).

\section{SCI SEVERITY}

One patient was categorized as being in ASIA B (Braddom, 2007). The rest of patients had complete motor SCI (ASIA A).

The final results of spasticity and ROM change, also electrophysiologic tests performed 1 year after finishing the study protocol were presented here. We also measured all these parameters immediately after finishing study protocol ( 2 months after beginning of the study). However, as the results of 1 year follow up was the same as the results obtained immediately after completing 2 month study protocol, we reported the results of 1 year follow up.

\section{SPASTICITY SCALE}

In Group 1 who did not use the EPPS at all, there was shown no significant change in the mean Ashworth spasticity scale before and after launching the EPPS. Meanwhile, in Group 2 who had used the EPPS up to the optimal level, the mean Spasticity Scale before application of EPPS was more than the mean Spasticity Scale after EPPS usage $(P=0.003)$.

\section{PASSIVE ROM}

In the group who did not use EPPS at all (Group 1) there was found no significant difference in mean ROM of hip (Add., Abd., Flex., Ext., Internal, and External Rotation), knee flexion, ankle dorsiflexion, and plantar flexion.

Finally, in Group 2 who used EPPS on an optimal basis, except for knee flexion, there was shown significant increase for ROM of hip (Abd, Add., Flex, and Ext), ankle dorsiflexion and plantar flexion (Table 2).

\section{ELECTRODIAGNOSTIC DATA}

In both intervention and control group, there was no significant difference in H-Reflex amplitude either before or after launching the EPPS.

There was a significant difference in decreasing $\mathrm{H}$ max/M max (RT\&LT) and F/M ratio after versus before exercising with the electric passive cycling system among the veterans who had exercised to the target level (Table 3 ).

Nevertheless, regarding other measured variables, there was no remarkable difference between those two groups.

\section{DISCUSSION}

Mean age for Iranian veterans participated in this study was 4045 years old, which is considered young likewise other countries that SCI primarily affects young men (Braddom, 2007; Frontera, 2008).

In our study, the most frequent level of injury was thoracic level. Hence; almost all of the SCI veterans in our study suffered from

Table 1| Motor and sensory distribution of the right and left spinal injury in SCI Veterans who were recruited in the study on EPPS application.

\begin{tabular}{|c|c|c|c|c|c|c|c|c|}
\hline \multirow[t]{3}{*}{ Spinal regions } & \multicolumn{4}{|c|}{ Right } & \multicolumn{4}{|c|}{ Left } \\
\hline & \multicolumn{2}{|c|}{$\begin{array}{l}\text { Motor region of } \\
\text { the spinal injury }\end{array}$} & \multicolumn{2}{|c|}{$\begin{array}{l}\text { Sensory region of } \\
\text { the spinal injury }\end{array}$} & \multicolumn{2}{|c|}{$\begin{array}{l}\text { Motor region of } \\
\text { the spinal injury }\end{array}$} & \multicolumn{2}{|c|}{$\begin{array}{l}\text { Sensory region of } \\
\text { the spinal injury }\end{array}$} \\
\hline & No. & $\%$ & No. & $\%$ & No. & $\%$ & No. & $\%$ \\
\hline Cervical & 11 & 17.2 & 8 & 12.5 & 12 & 18.8 & 8 & 12.5 \\
\hline Upper thoracic & 22 & 34.4 & 20 & 31.3 & 21 & 32.8 & 20 & 31.3 \\
\hline Lower thoracic & 29 & 45.3 & 28 & 43.8 & 28 & 43.8 & 28 & 43.8 \\
\hline Lumbar & 2 & 3.1 & 2 & 3.1 & 2 & 3.1 & 1 & 1.6 \\
\hline Unidentified & 0 & 0 & 0 & 0 & 1 & 1.6 & 7 & 10.9 \\
\hline Total & 64 & 100 & 64 & 100 & 64 & 100 & 64 & 100 \\
\hline
\end{tabular}


Table 2 | The comparison between mean ROM of joints in Lower limbs before and after receiving the EPPS protocol in veterans used the EPPS optimally.

\begin{tabular}{llrrl}
\hline \multicolumn{1}{c}{ Variant } & & Mean value & SD & $P$ value \\
\cline { 2 - 5 } Hip abd. ROM & Before EPPS & 18.9655 & 9.3902 & 0.003 \\
& After EPPS & 23.1034 & 7.6080 & \\
Hip add. ROM & Before EPPS & 15.8621 & 7.3276 & 0.005 \\
& After EPPS & 18.9655 & 5.5708 & \\
Hip flex. ROM & Before EPPS & 85.3448 & 22.2779 & 0.000 \\
& After EPPS & 103.7931 & 13.9933 & \\
Hip ext. ROM & Before EPPS & 7.4138 & 7.8627 & 0.000 \\
& After EPPS & 12.0690 & 6.1986 & \\
Knee flex ROM & Before EPPS & 89.3103 & 28.0218 & 0.111 \\
& After EPPS & 150.000 & 204.7646 & \\
Ankle dorsiflex ROM & Before EPPS & 3.5714 & 5.4189 & 0.000 \\
& After EPPS & 13.5714 & 6.2148 & \\
Ankle plantar flex & Before EPPS & 9.8214 & 10.2227 & 0.000 \\
& After EPPS & 19.2857 & 10.8622 & \\
& & & &
\end{tabular}

According to the table, apart from knee flexion, mean difference in the rest ROM was significant $(P<0.05)$.

Table 3 | Comparison between mean value of LT H $(\max ) / \mathrm{M}(\max )$ and RT H (max)/M (max), before and after EPPS application in veterans who conducted the pattern of optimal exercise with EPPS.

\begin{tabular}{llll}
\hline Variables & Mean value & SD & $P$ value \\
\hline RT H (max)/M (max) 1 & 0.3984 & 0.1902 & 0.000 \\
RT H (max)/M (max) 2 & 0.1963 & 0.1348 & \\
LT H $(\max ) / M(\max ) 1$ & 0.4022 & 0.1828 & 0.000 \\
LT H $(\max ) / M(\max ) 2$ & 0.1989 & 0.1176 & \\
RTF/M ratio 1 & 0.2063 & 0.2631 & 0.027 \\
RTF/M ratio 2 & 0.0923 & 0.1399 & \\
LTF/M ratio 1 & 0.2036 & 0.2697 & 0.025 \\
LTF/M ratio 2 & 0.0717 & 0.1043 & \\
\end{tabular}

1, Before exercise; 2, after exercise.

mostly complete lower limbs disability. According to international references, cervical spinal cord injuries occur more frequently than thoracic and lumbar injuries (Frontera, 2008).

The next issue is that, most of the veterans had been injured when the Iran-Iraq war was on its prime days, but they developed SCI complications gradually during 15-20 years after their primary injury at war days.

We assessed mainly objective signs in this study and it is to be emphasized that considering objective signs is significant because it crosses out the interference from patients, leading to the measurement of interventional factors and shows their crucial effect.

\section{RANGE OF MOVEMENT}

The ROM for lower limbs are of high significance in SCI veterans health condition since long term sedentary life leads to comparative stiffness in the joints (McArdle et al., 1994; Braddom, 2007; Frontera, 2008). Gently loosening and increasing ROM in these joints are the ultimate goals of rehabilitation in these veterans and if sustained it will be an immense contribution to them.

In our study, in veterans who used EPPS on an optimal basis, significant increase for ROM of hip, ankle dorsiflexion, and plantar flexion were noticed, indicating effective role of EPPS on ROM of these joints.

However, no change in knee flexion ROM was noticed. There could be an explanation for that finding. In patients with optimum knee flexion from the very beginning of the trial, no alteration in ROM values was noticed and it was due to their original proper knee flexion. While those without proper knee flexion probably could not use EPPS properly and obtain appropriate results from exercising with EPPS.

In similar studies conducted to determine the effects of passive leg cycle exercise in patients with SCI, increased muscle force and torque output were observed in SCI patients (Hjeltnes et al., 1997; Scremin et al., 1999). In another study, exercise endurance, pedal revolutions per minute ( $\mathrm{rpm}$ ) and peak power output were improved after 8 weeks of training (3 days/week; Priest et al., 1995; Krause et al., 2008).

Long term FES cycling was shown to enhance fitness, decrease insulin resistance, and increase mass in persons with SCI (Braddom, 2007), it can improve quadriceps muscle strength (Therese et al., 2008), and to a lesser extent, cycling power output significantly (Duffell et al., 2008; Therese et al., 2008).

Even though there are numerous studies evaluating effect of FES on muscle properties of SCI people, less research has focused on the benefits of passive cycling in individuals with SCI (Kakebeeke et al., 2005; Therese et al., 2008).

The mean spasticity scale in veterans who practiced with the pedaling device to the target level or below the target showed significant decrease in comparison with the spasticity scale before providing the electric passive cycling system. This is to show the significant effect of exercise with EPPS in decreasing the spasticity.

Passive rhythmic activities reported to improve Spasticity (Katz, 1991; Nuyens et al., 2002; Sköld et al., 2002). FES propelled cycling lead to reduced spasticity in SCI subjects who achieved smooth pedaling in different studies (Hoffman, 1986; Katz, 1991; Krause et al., 2008; Szecsi and Schiller, 2009). In a study by Rösche et al. (1997) a motorized exercise-cycle was also used to treat spasticity in subjects who suffered predominantly from multiple sclerosis. However, in two other experiments, no significant reduction in spasticity with Ashworth scale was noticed after rhythmic passive movements of the legs in SCI subjects. However, subjectively, the patients profited from the cycling (Kakebeeke et al., 2005; Therese et al., 2008).

Regarding Electrodiagnostic findings, EPPS had no effects on reduction of $\mathrm{H}$-Reflex and $\mathrm{F}$-wave amplitudes in our study. This is against Rösche's findings through his research. In his study, mean F-wave amplitude, mean F-wave/M-response ratio, and maximum F-wave/M-response ratio were significantly lower after utilizing a motorized exercise-cycle than before. Though it is to be emphasized that he had not evaluated the usage of EPPS for a long period of time (Rösche et al., 1997), while in the current study the effect of EPPS on F-wave amplitude was evaluated after 1 year period and no reduction in F-wave amplitude was demonstrated. However, like Rösche's study, mean F/M wave and H/M reflex was 
decreased after cycling. Clinically this electrophysiologic change can be reflected in reducing spasticity related to loss of supraspinal inhibitory mechanisms noticed in SCI patients. In Rösche's study, it was suggested that F-wave amplitudes can be used to show changes of motor neuron excitability in patients receiving antispastic therapy and document the effect of the therapy with motorized passive cycling.

Spinal cord injury subjects are prone to cardiovascular disorders leading to increased risk of mortality, due to their physical inactivity and dependency for mobility (Nuyens et al., 2002; Ballaz et al., 2007). Passive leg cycle exercise was shown to have positive effects on vascular blood flow velocity (Ballaz et al., 2007), also oxygen uptake (VO2). Regular lower leg exercise can result in significant gains not only in physical, but also psychological wellbeings (Lannem et al., 1990; Hicks et al., 2003; Hjeltnes, 2009). Nowadays, there are also commercially available powered cycle trainers that subjects can use at home (Therese et al., 2008).

In conclusion, We found beneficial physical effect of EPPS in our study represented in electrodiagnostic and other objective measures in SCI veterans. The spasticity level was reduced and passive ROM were also improved in SCI veterans used EPPS

\section{REFERENCES}

Ashworth, N. L., Satkunam, L. E., and Deforge, D. (2004). Treatment for spasticity in amyotrophic lateral sclerosis/motor neuron disease. Cochrane Database Syst. Rev. 1, CD004156.

Ballaz, L., Fusco, N., Crétual, A., Langella, B., and Brissot, R. (2007). Acute peripheral blood flow response induced by passive leg cycle exercise in people with spinal cord injury. Arch. Phys. Med. Rehabil. 88, 471-476.

Braddom, R. L. (2007). Physical Medicine and Rehabilitation, Chapter 56, 3rd Edn. Philadelphia: Saunders Elsevier, 1286-1349.

Daivis, G. M. (1993). Exercise capacity of individuals with paraplegia. Med. Sci. Sports Exerc. 25, 423-432.

Devillard, X., Rimaud, D., Roche, F., and Calmels, P. (2007). Effects of training programs for spinal cord injury. Ann. Readapt. Med. Phys. 50, 490-498, 480-489.

Duffell, L. D., Donaldson, Nde N., Perkins, T. A., Rushton, D. N., Hunt, K. J., Kakebeeke, T. H., and Newham, D. J. (2008). Long-term intensive electrically stimulated cycling by spinal cord-injured people: effect on muscle properties, and their relation to power output. Muscle Nerve 38, 1304-1311.

Fierro, B., Raimondo, D., and Modica, A. (1990). Analysis of F response in upper motoneurone lesions. Acta. Neurol. Scand. 82, 329-334.
Fox, J. E., and Hitchcock, E. R. F. (1987). F wave size as a monitor of motor neuron excitability: the effect of deafferentation. J. Neurol. Neurosurg. Psychiatr. 50, 453-459.

Frontera, W. (2008). Essential of Physical Medicine and Rehabilitation, Chapter 146-148, 2nd Edn. Saunders Elsevier, 853-879.

Hicks, A. L., Martin, K. A., Ditor, D. S., Latimer, A. E., Craven, C., Bugaresti, J., and McCartney, N. (2003). Long-term exercise training in persons with spinal cord injury: effects on strength, arm ergometry performance, and psychological well-being. Spinal Cord 41, 34-43.

Hjeltnes, N. (2009). Incomplete spinal cord injury, exercise and life satisfaction. Spinal Cord 47, 295-300.

Hjeltnes, N., Aksnes, A. K., Birkeland, K. I., Johansen, J., Lannem, A., and Wallberg-Henriksson, $\mathrm{H}$. (1997). Improved body composition after $8 \mathrm{wk}$ of electrically stimulated leg cycling in tetraplegic patients. Am. J. Physiol. 273, RI072-R1079.

Hoffman, M. D. (1986). Cardiorespiratory fitness and training in quadri3, 312-330.

Kakebeeke, T. H., Lechner, H. E., and Knapp, P. A. (2005). The effect of passive cycling movements on spasticity after spinal cord injury: preliminary results. Spinal Cord 43, 483-488. plegics and paraplegics. Sports Med.

at optimal level. Furthermore, these positive effects 1 year after passive cycling was stopped, was similar to the results obtained immediately after 2 month passive cycling. Our results demonstrated that patients using EPPS gained better results compared with manual passive movements. EPPS is a method of achieving continues and regular passive movement, but stretching parameters like speed or smoothness of passive movement may not be controlled by manual stretching. We suggest that response to electrical passive cycling can be monitored via measuring electrophysiological parameters besides clinical spasticity measurement scales (Ashworth's scale, etc.). Monitoring motor neuron excitability via measuring electrophysiologic parameters was also indicated in previous studies (Fox and Hitchcock, 1987; Fierro et al., 1990; Rösche et al., 1996, 1997). According to our participants, EPPS did not induce discomfort. Instead, they mentioned verbally a sensation of well-being. However, further work should address the other aspects of the outcomes of long term Electrical Passive Cycling in SCI veterans.

We suggest to conduct more studies with larger samples for evaluating the effect of passive cycling on spasticity, ROM and other muscle properties.

Katz, R. T. (1991). Management of spastic hypertonia after stroke. J. Neuro. Rehab. 5, S5-S12.

Krause, P., Szecsi, J., and Straube, A. (2008). Changes in spastic muscle tone increase in patients with spinal cord injury using functional electrical stimulation, and passive leg movements. Clin. Rehabil. 22, 627-634.

Lannem, A. M., Fierro, B., Raimondo, D., and Modica, A. (1990). Analysis of $\mathrm{F}$ response in upper motoneurone lesions. Acta. Neurol. Scand. 82, 329-334.

McArdle, W. D., Katch, F. I., and Katch, V. L. (1994). Essentials of Exercise Physiology, 2nd Edn. Philadelphia, PA: Lippincott Williams \& Wilkins, c2000.

Mutton, D. L., Scremin, A. M., Barstow, T. J., Scott, M. D., Kunkel, C. F., and Cagle, T. G. (1997). Physiologic responses during functional electrical stimulation leg cycling and hybrid exercise in spinal cord injured subjects. Arch. Phys. Med. Rehabil. 78, 712-718.

Nuyens, G. E., De Weerdt, W. J., Spaepen, A. J. Jr., Kiekens, C., and Feys, H. M. (2002). Reduction of spastic hypertonia during repeated passive knee movements in stroke patients. Arch. Phys. Med. Rehabil. 83, 930-935.

Priest, J., Hagan, R., Simpson, S., and Jennings, R. (1995). Improved exercise capacity of paraplegics during unassisted leg cycling on an energystorage ergometer. Med. Sci. Sports Exert. 27, S137.
Rösche, J., Paulus, C., Maisch, U., Kaspar, A., Mauch, E., and Kornhuber, H. H. (1997). The effects of therapy on spasticity utilizing a motorized exercise-cycle. Spinal Cord. 35, 176-178.

Rösche, J., Riib, B., Niemann Delius, B., Mauch, E., and Kornhuber, H. H. (1996). Effects of physiotherapy on F waves amplitudes in spasticity. Clin. Neurophysiol. 36, 509-511.

Scremin, A. M., Kurta, L., Gentili, A. Wiseman, B., Perell, K., Kunkel, C., and Scremin, O. U. (1999). Increasing muscle mass in spinal cord injured persons with a functional electrical stimulation exercise program. Arch. Phys. Med. Rehabil. 80, 1531-1536.

Sköld, C., Lönn, L., Harms-Ringdahl, K., Hultling, C., Levi, R., Nash, M. and Seiger, A. (2002). Effects of functional electrical stimulation training for six months on body composition and spasticity in motor complete tetraplegic spinal cord-injured individuals. J. Rehabil. Med. 34, 25-32.

Szecsi, J., and Schiller, M. (2009). FES-propelled cycling of SCI subjects with highly spastic leg musculature. NeuroRehabilitation 24, 243-253.

Therese, E., Johnston, T. E., Smith, B. T., Mulcahey, M. J., Betz, R. R., and Lauer, R. T. (2009). A randomized controlled trial on the effects of cycling with, and without electrical stimulation on cardiorespiratory, and vascular health in children with 
spinal cord injury. Arch. Phys. Med. Rehabil. 90, 1379-1388.

Therese, E., Johnston, T. E., Smith, B. T., Oladeji, O., Betz, R. R., and Lauer, R. T. (2008). Outcomes of a home cycling program using functional electrical stimulation or passive motion for children with spinal cord injury: a case series. J. Spinal Cord Med. 31, 215-221.
Conflict of Interest Statement: The authors declare that the research was conducted in the absence of any commercial or financial relationships that could be construed as a potential conflict of interest.

Received: 31 January 2011; accepted: 07 June 2011; published online: 20 June 2011.
Citation: Rayegani SM, Shojaee $H$, Sedighipour L, Soroush MR, Baghbani $M$ and Amirani $O B$ (2011) The effect of electrical passive cycling on spasticity in war veterans with spinal cord injury. Front. Neur. 2:39. doi 10.3389/fneur.2011.00039

This article was submitted to Frontiers in Spinal Cord Medicine, a specialty of Frontiers in Neurology.
Copyright (c) 2011 Rayegani, Shojaee, Sedighipour, Soroush, Baghbani and Amirani. This is an open-access article subject to a non-exclusive license between the authors and Frontiers Media $S A$, which permits use, distribution and reproduction in other forums, provided the original authors and source are credited and other Frontiers conditions are complied with. 